\title{
Age at Deafening Affects the Stability of Learned Song in Adult Male Zebra Finches
}

\author{
Anthony J. Lombardino and Fernando Nottebohm \\ The Rockefeller University, Field Research Center for Ecology and Ethology, Millbrook, New York 12545
}

\begin{abstract}
Male zebra finches (Taeniopygia guttata) master the imitation of a song model 80-90 d after hatching and retain it with little change for the rest of their lives. Acquisition and maintenance of this imitation require intact hearing. A previous report showed that male zebra finches deafened as adults start to lose some of the acoustic and temporal features of their song a few weeks after deafening and that by 16 weeks the learned song is severely degraded (Nordeen and Nordeen, 1992). However, this previous study noted no correlation between the age at deafening and the subsequent timing and extent of song loss. We deafened adult male zebra finches ranging in age from $81 \mathrm{~d}$ to 6 years. The song of birds deafened at the younger ages
\end{abstract}

Song learning in oscine songbirds provides tantalizing parallels to speech learning in humans (Marler, 1970). Both involve inherited predispositions for learning (Gould and Marler, 1987), both require intact auditory feedback for acquisition of the learned pattern, and both go through early stages-subsong in birds and babbling in humans-that are thought to constitute a form of vocal "play" or practice that precedes the serious business of imitation (Thorpe, 1958).

Little is known about how learned vocalizations, after being acquired, are maintained. In both adult humans and some songbirds, auditory feedback has been thought to guide the acquisition of learned sounds but to play less of a role in maintaining them (Konishi, 1965; Borden, 1979). It has been suggested recently that the severity of the effects of sudden deafness on speech depends on the age at onset of deafness (Waldstein, 1990). The agedependent differences reported by this author resulted from comparing the effects of onset of deafness in children versus adults. We here present observations on birds that fit well with the scarce data on humans and that extend the effect of age well into adulthood. As with acquisition, there may also be striking parallels in the mechanisms that underlie the maintenance of learned vocal skills in humans and songbirds.

Zebra finch males reared in a captive colony master the imitation of adult models by the time they reach sexual maturity, 80-90 d after hatching (Immelmann, 1969; Arnold, 1975a; Price, 1979;

\footnotetext{
Received Dec. 21, 1999; revised April 10, 2000; accepted April 10, 2000.

This work was supported by Public Health Service (PHS) Training Grant GM 07524 and PHS Grant MH 18343. We are grateful to Marta E. Nottebohm and Uwe Hahnman for scoring sonagrams; David Vicario, Constance Scharff, and Anthony Tramontin for their helpful comments on the text; Robert Suter for advice on statistical analysis; Ofer Tchernichovski for assistance with the Song Analysis software; and Daun Jackson, Sharon Sepe, and Helen Ecklund for their outstanding bird care.

Correspondence should be addressed to Dr. Anthony Lombardino, Rockefeller University Field Research Center, Tyrrel Road, Millbrook, NY 12545. E-mail: lombara@rockvax.rockefeller.edu.
}

Copyright (C) 2000 Society for Neuroscience $\quad 0270-6474 / 00 / 205054-11 \$ 15.00 / 0$
(81-175 d) deteriorated severely after a few weeks, and within that age bracket, the older the bird was at deafening, the longer it took for this degradation to occur and the slower the subsequent process of song deterioration. The song of birds deafened at 2 years and older showed little change during the first 51 weeks after deafening but was grossly altered by 100 weeks. We suggest (1) that this age effect could be independent of experience or (2) that each time a bird sings, a little bit of learning - motor engrainment-occurs, adding to memory duration in a cumulative manner.

Key words: song system; vocal learning; zebra finch; deafness; auditory experience; motor memory
Böhner, 1990; Volman and Khanna, 1995). This conversion of an auditory model into a motor replica occurs only once, during the so-called "sensitive period" for song learning (but see Eales, 1985, 1987; Morrison and Nottebohm, 1993). Thereafter, song changes very little. For this reason zebra finches are referred to as sensitive-period learners.

However, song learning may not end then. Nordeen and Nordeen (1992) showed that the song of male zebra finches deafened several months after they had acquired their adult song did, nonetheless, deteriorate. In that sample there was no interaction between the age at deafening and subsequent song deterioration among individuals ranging in age between 120 and $430 \mathrm{~d}$. We here show, using a wider range of adult ages, that song deterioration after deafening starts much sooner and proceeds more quickly in young adults than in older adults. We infer that the status of the circuits that control the production of a learned motor skill is very sensitive to age or to amount of experience. This raises some interesting questions about the mechanisms that underlie the long-term maintenance of a learned motor skill.

\section{MATERIALS AND METHODS}

Subjects. Our 19 subjects were the offspring of a captive colony of 10 breeding pairs housed in an indoor aviary at The Rockefeller University Field Research Center in Millbrook, NY. Sixteen of these birds, ranging in age from $81 \mathrm{~d}$ to $>5$ years after hatching, were selected for surgery; this total includes one bird in which the deafening operation was done in two steps, with one cochlea removed on each of 2 separate days. The remaining 3 unoperated birds were recorded at two widely disparate times to serve as a control for normal changes to song over time. In addition, 3 of the birds that were later deafened also served, during their first 5-6 years, as controls for normal changes to song over time, yielding a total $n$ of 6 intact controls. All 19 birds were housed singly in cages that were close to others in the same room also holding zebra finches. To encourage song production, the position of the single cages was changed every few weeks so that birds never had the same neighbor for long periods of time. Dry seed and water were provided ad libitum, and a mixture of freshly sprouted seed and ground egg with vitamins (Avia; Nutra-Vet Research Corporation, Poughkeepsie, NY) was provided 
daily. All birds were kept at all times on a 12:12 hr light/dark photoperiod.

Age groups. Our 16 deafened birds were assigned to one of three age groups, which were set up by reference to the age at deafening. The "young" group included 6 birds deafened between the ages of 81 and $137 \mathrm{~d}$ after hatching. The "middle-aged" group included 3 birds deafened at 169-231 d. The "old" group included 7 birds deafened at 664-2090 d. The middle-aged group corresponded to the age at which other workers had deafened their zebra finches and for this reason was set up as a separate group.

Recordings of song and calls. Birds were recorded several days before surgery with a Marantz PMD221 portable cassette recorder on Maxell XLIIS magnetic tape. Long calls and song were recorded and used as the behavioral data. Both kinds of vocalization are learned (Immelmann, 1969; Price, 1979; Zann, 1985) and controlled by the forebrain motor pathways for production of learned sounds (Simpson and Vicario, 1990).

Male zebra finches presented with females court them (see Morris, 1954; Walters et al., 1991), and part of this courtship is "directed" song, i.e., song produced at close range while the male adopts a typical posture and faces the female. This directed song can also be given to another male, also at close range. An almost identical pattern of song, however, can be produced by males placed in a room by themselves, and then it is referred to as "undirected" song (Immelmann, 1969). Differences between directed and undirected song are subtle and do not involve changes in the structure or order of song syllables (Bischof et al., 1981; Böhner et al., 1992). Recordings of intact birds used only directed song, but our postoperative recordings included both directed and undirected song, and the effects of deafening that we report here are not exclusive to songs delivered in either context. The number of songs (motifs, see below) recorded and examined for each bird at any one sampling period varied, ranging from 15 to 25 . From these we drew the most complete and clear recording, which therefore best represented the bird's singing ability at that time. Whenever possible, our analysis used recordings of directed song, but when birds did not sing in this mode, they were placed singly in a cage, and a voice-activated system (with digital delay and amplitude detection circuit) recorded their undirected song.

All birds that were deafened were recorded again within days of surgery and periodically (every 1-3 weeks) thereafter for up to 51 weeks. If song at 51 weeks after the operation still showed little or no change, two subsequent recordings were taken at $\sim 70$ weeks and at $100-110$ weeks after deafening.

Surgery. Subjects were deafened by cochlear extirpation, as described (Konishi, 1964, 1965). Briefly, after the bird was anesthetized with Nembutal, a small window was made through the back of the skull overlying the semicircular canals. A small hole was then made in the cochlear dome, and a fine, hooked wire was lowered into this hole and used to pull out the cochlea. Removal of each cochlea was confirmed by visual inspection under the dissecting microscope. To control for possible nonspecific effects of the surgery, one young adult bird had only a single cochlea removed. This bird's vocalizations were recorded for several months, and the second cochlea was then removed, with recordings taken up to 60 weeks thereafter. Input from each cochlea reaches both hemispheres (Kelley and Nottebohm, 1979), which therefore should continue to receive song feedback after unilateral cochlear removal.

In some birds, an alternative approach was taken to remove the cochleae; under anesthesia, a hole was made in the tympanic membrane, and the columella was extracted with a forceps. The hooked wire was then inserted through the oval window, and the cochlea was pulled out. There were no obvious differences in outcome between these two operations.

Sound analysis. Song has complexity on several time scales, and there are many acoustic features that may be of interest, either in intact or deafened birds. Some features are readily and reliably measured by computer; others can be evaluated quite effectively by human scorers. We chose to measure several features of intact song by computer to assess its stereotypy. These stereotypy measurements, gleaned from recordings that lasted a few minutes, were done in birds of different ages. We did not use the same computer program to assess song changes over longer periods of time (days or months), because the recording conditions often changed over these longer periods and resulted in the inclusion of more or less background noise, which interfered with the computer's "blind" approach. For comparisons over these longer periods, we used human raters that did not know to which group each bird belonged.

Our use of terminology to describe the components of adult zebra finch song is shown in Figure 1. The song of adult male zebra finches
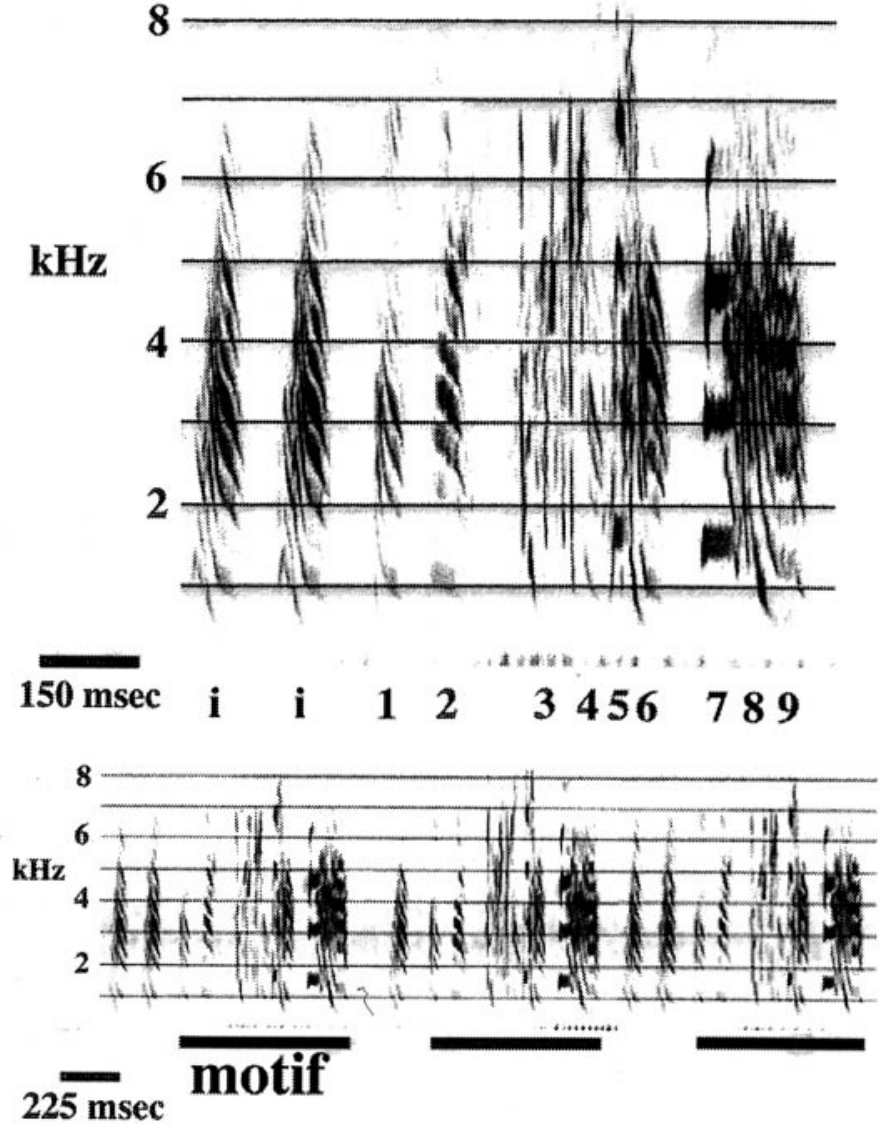

Figure 1. Typical zebra finch song structure, as revealed by soundspectrographic analysis. Top, The motif is usually preceded by an introductory note (labeled $i$ ) repeated a variable number of times. The motif itself consists of a series of discrete sounds, usually delivered in the same order, that we call syllables and that are numbered here 1-9. Syllables have a distinct acoustic structure, with a beginning and end marked by a silent interval, as in syllables 1 and 2, or by an abrupt change in frequency modulation, as in syllables 7-9. Clusters of syllables (e.g., syllables 3-6 and 7-9) separated from previous and subsequent sounds by a longer silent interval $(>10 \mathrm{msec})$ are called chunks. The motif is usually repeated a number of times, forming a bout (see Fig. 9). Bottom, Repetitions of the motif, shown here at the beginning of three successive song bouts, are presented. Notice that with the exception of the variability in the number of introductory notes, the fine structure of the motif is highly stereotyped.

consists of a series of "syllables." Each syllable is a continuous trace on a sound spectrogram, separated from adjacent syllables by either silence or a rapid change in frequency modulation (Sossinka and Böhner, 1980; Williams, 1990). Syllables have been shown to be a natural unit of song patterning (Cynx, 1990). Groups of syllables preceded and followed by a longer silent interval (typically $30-40 \mathrm{msec}$ and at least $10 \mathrm{msec}$ ) form a "chunk" (Williams and Staples, 1992). Several chunks, produced in a fixed order, constitute a "motif." Each adult male zebra finch has a single motif, which is usually preceded by introductory notes and repeated several times forming a song "bout." The order of syllables and chunks within the motif and its overall duration are very stereotyped features in zebra finch song (Scharff and Nottebohm, 1991), as shown in Figure 1.

Tape recordings were converted to sonagrams on a Kay Sonagraph (model 7800) using the $300 \mathrm{~Hz}$ (wide-band) filter setting. To quantify the changes to song and long calls after deafening, two human raters (neither of the authors) blind to the experimental group to which each bird belonged but familiar with zebra finch song and its display by sonagram scored the printed sonagrams. The visual inspection technique makes use of the potent pattern recognition capabilities of the human visual system. These pattern recognition capabilities have been shown to recognize similarities and differences in songs in a manner comparable with other, more quantitative, sound spectrograph-based techniques (Nowicki and Nelson, 1990; Marler and Nelson, 1993). 
Evaluating the song of intact birds. Stereotypy is a defining feature of adult zebra finch song (Scharff and Nottebohm, 1991; Zann, 1993), and we looked at this feature in intact birds of different adult ages as a possible predictor of the rate of song degradation after deafening. This point is of particular interest with the youngest deafened birds because it could be argued that, for example, birds 80-90 d old were not really adults yet and that their song was still unstable.

We used four measures to quantify song stereotypy in intact birds. The first two involved the traditional method used with zebra finch song: sequence linearity (SL) and sequence consistency (SC), as defined by Scharff and Nottebohm (1991). SL, which refers to the way syllables are ordered in a song, is expressed as the number of syllable types per number of different transition types present in a sample of song. In a completely linear song sequence, each syllable has only one transition type; that is, it is followed by only one other syllable (or motif end), and the SL ratio is equal to 1 . In practice, sometimes a syllable is omitted or could occur in the "wrong" order, in which case an atypical transition occurs and the SL ratio becomes $<1$. SC addresses how often the typical transitions occur. SC is expressed as the sum of typical transitions per the sum of all transitions per song sample. For both our SL and SC calculations we considered the transition between the last introductory note and the first motif syllable and the transition between the last motif syllable and the end of song as part of the transitions counted. We computed our estimates of SL and SC for a total of 16 intact birds ranging in age from $81 \mathrm{~d}$ to $>5$ years after hatching, using 20 motif repetitions per bird. The SL and SC scores for birds of different ages were compared statistically using a two-tailed Mann-Whitney $U$ test.

The third method we used to assess stereotypy of intact song was to measure the consistency with which birds of various ages sang a chunk of their song. Exemplars of intact song from 16 birds were digitized using the software package Canary (Cornell Laboratory of Ornithology, version 1.2.1). The duration of the chunks was measured for 15 repetitions of a motif, obtained from several song bouts. The SD of these measurements was determined for each bird, and a two-tailed $t$ test was performed on these values to assess the significance of differences between the two age groups.

Finally, in an effort to score our songs more analytically, we used a fully automated song comparison procedure described recently by Tchernichovski et al. (2000) to assess intact-song stereotypy. This procedure compares two digitized sounds on the basis of four features: Wiener entropy, spectral continuity, pitch, and frequency modulation. This procedure was developed, initially, to compare the degree of similarity between the song of a tutor and that of its pupil. In this context, measures of overall similarity between songs using visual scoring by humans and that by the automated algorithm are comparable (Tchernichovski et al., 2000). However, the automated algorithm is better suited for measuring the degree of variability that might be present in successive repetitions of a same-song motif, which is why we used it to obtain a measure of stereotypy. We did this in 21 intact adults of various ages. This was done by getting digitized samples of $16-25$ motif repetitions per bird, obtained from at least three different song bouts, and comparing each repetition with all others. There were no systematic differences in sample size between the age groups. An average of 159 comparisons were performed for each bird (range, 91-300; total for all birds = 3341). The percent similarity score (a measure of stereotypy) for any one bird on any one day was the mean of these comparisons.

Evaluating the effects of deafening. Changes that occurred after deafening were much more marked than the variability we saw in the intact birds, and to quantify these changes we used methods that were different from the ones used to gauge song stereotypy in intact birds. Scorers were given code-labeled sonagrams with representative songs recorded before surgery and at some time point after surgery. For these comparisons we used sound spectrograms that were particularly crisp and free of echoes or background noise and that included the most complete array of syllables that formed a motif. The scorers were asked to find in each postoperative recording the closest match to each syllable in the presurgery song. Scorers were explicitly told to use sequence information (the relative position of syllables) to identify homologous preoperative and postoperative syllables; the sequence of syllables is usually very fixed in adult song (Sossinka and Böhner, 1980; Williams and Staples, 1992). They then scored the degree of similarity using the following scale: 0 , no syllable in the postsurgery song even slightly similar to a given presurgery syllable; 1 , slight similarity; 2 , moderate similarity; 3 , strong similarity; and 4 , a virtually perfect match between the presurgery and postsurgery syllables. The scores for all syllables in a single song were averaged to give a measure of the overall similarity of the presurgery and postsurgery songs, referred to as the song similarity index (SSI). The mean scores $(n=57)$ produced by each of the two scorers were very close (correlation coefficient $=0.95$ ). Because the corresponding values obtained by the two scorers were so similar, the two scores obtained for each time point after deafening were averaged. We also obtained SSI scores for the song of intact birds recorded over intervals of 2-5 years, so that the effects of aging, per se, could be separated from the effects of deafening at various postsurgery intervals. The Mann-Whitney $U$ test (one-tailed, because birds could only have lower SSIs after deafening) was used to compare the mean SSIs of all intact control birds with the SSIs of the deafened birds. This comparison erred in the direction of conservatism in that the time spans involved in the intact birds were longer than those involved in the comparison of presurgery and postsurgery songs in the deafened birds. For the latter comparison the young and the middle-aged birds were lumped together as one group, and the old birds formed another group.

The similarity scores described above take into account the overall structure, frequency, and position of syllables, without singling out specific features, and are similar to a "Gestalt" comparison. To obtain a finer-grained measure of song degradation, we measured the duration of clearly discernible chunks of song, identified either by silent intervals on either side or by homology to previous recordings, preoperatively and at various later intervals. These measurements took advantage of a spectral derivative visual display of song (Tchernichovski et al., 2000) to visualize chunks. The time span over which this analysis could be done depended very much on the rate of deterioration of song structure, which was particularly steep in the young birds. That is, the duration of chunks, as data, made sense only if chunks were well defined. In most (but not all) of the young birds, chunk duration measurements were feasible during the first 10-20 weeks after deafening. In the older birds, the same measurements could be done up to 100 weeks after deafening. Quantitative comparisons of the stability of chunk duration in all three age groups was restricted to the first 8 weeks after deafening, because for that period we had data for all birds in our three age groups. We also present graphically exemplars of the time course of the change in chunk duration in one representative young and one representative old bird (see Fig. 7).

\section{RESULTS}

\section{Long-term stability of song in adult intact zebra finches}

Figure 2 shows examples of the stability of song and long calls in adult male zebra finches recorded over a 4-5 year interval. Although Immelmann (1969) reported that zebra finches maintained their song for up to 5 years, the one bird for which he published this information was cross-fostered to a Bengalese finch and thus was not singing its species-typical song. Here we show the more typical situation of zebra finches singing zebra finch song. These birds had been kept during the intervening years in cages with other zebra finches, often in breeding aviaries. Spontaneous modifications to directed song in these intact adults, as shown in this figure, included the deletion or addition of a short call (Fig. $2 B$ ) or long call (Fig. $2 C$ ) at the end of a motif, a change in harmonic emphasis (Fig. $2 A$ ), or a change in the duration of some syllables and of the silent intervals that separated them (Fig. $2 C$ ). These kinds of minor changes occurred on top of an overall lasting wealth of structure and order. These three birds were part of our intact control group, and more quantitative data will be given below when they are compared with our deaf birds.

\section{Same-day song stereotypy in intact young and old adults}

Our measures of stereotypy, obtained from recordings from a particular day, used exclusively directed song. Figure 3 shows two of our measures of stereotypy in young (81-111 d; $n=5)$ and old (931-2000 d; $n=6)$ adults. There were no statistical differences between age groups in scores for sequence linearity and sequence consistency (Fig. $3 A)\left(U_{\text {linearity }}=36 ; p>0.10 ; U_{\text {consistency }}=42\right.$; $p>0.10)$ or in the variability of chunk duration (Fig. $3 B)(t$ test, 


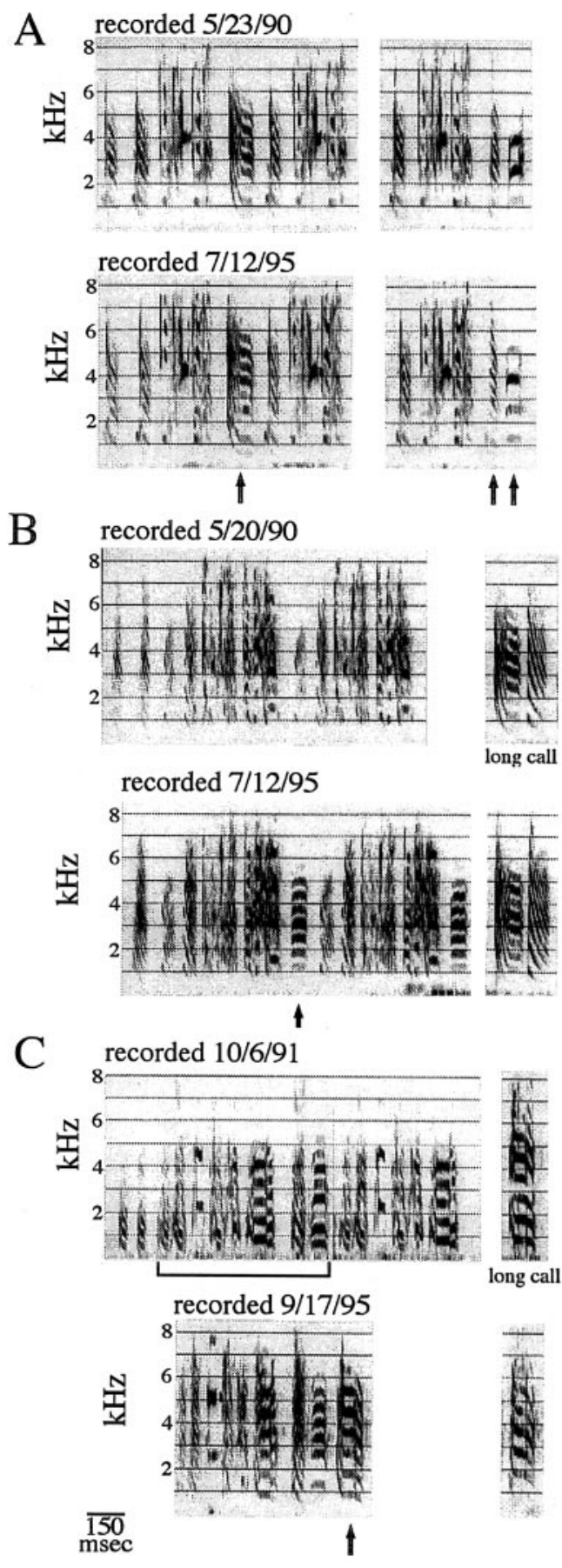

Figure 2. $A-C$, Song stability in three intact adult zebra finches over a long period of time. The long calls for two of these birds $(B, C)$ are also shown. The two renditions of the song or call of the same bird have been aligned vertically to facilitate comparison of temporal and acoustic features. Each bird was at least 6 months old at the time of the first recording. As shown by the dates printed above each song, 4-5 years elapsed between the first and second recordings shown. Overall, the learned vocalizations changed relatively little in time. However, notice that some flexibility in song production is possible even with a closed-ended learner such as the zebra finch. One of the birds $(A)$ produced two alternate, yet closely related, versions of the same motif, which differed in the last one or two syllables (arrows), an idiosyncrasy that persisted over the years. The kinds of changes that occurred over time were, for example, slight changes in harmonic emphasis (e.g., $A$, last syllable), the addition of the short call $(B$, arrow) or long call $(C$, arrow) at the end of the motif, and small changes in the temporal structure of the motif $(C$, notice the misalignment of the songs).
A

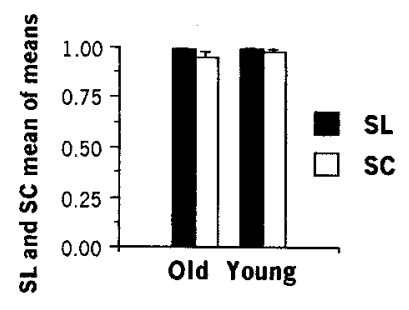

B

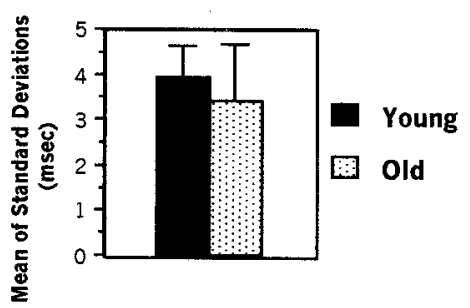

Figure 3. Quantification of song stereotypy in young (81-111 d of age) and old (931-2090 d of age) hearing adults. $A$, Mean of the means of SC and SL measures, based on 20 songs from each bird. Error bars correspond to SEs. $B$, Stereotypy of chunk durations. SDs were calculated from 15 repetitions of a chunk sung by a given bird; the vertical bars depict the mean of these eight SDs for each age group. Error bars are the SEs among birds in an age group. There was not a statistically significant difference between the groups.

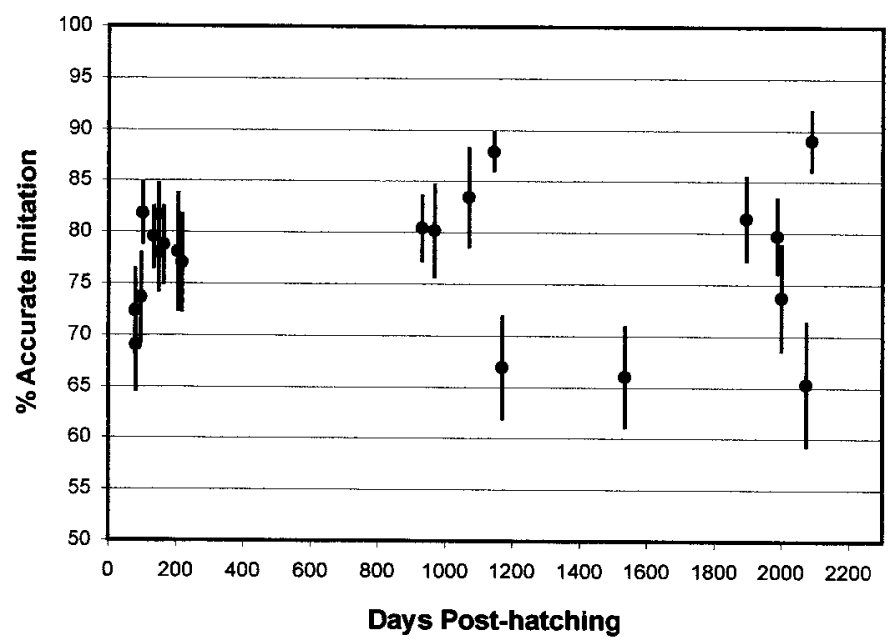

Figure 4. Graph of song stereotypy as a function of age in intact adults. Stereotypy here was measured using the song analysis algorithm developed by Tchernichovski et al. (2000) (see Materials and Methods). Each bird was scored on the variability it showed in repeating 15-25 renditions of its song motif. Had all repetitions been identical, the similarity score would have been $98-99 \%$. For each bird, each rendering of that bird's motif was compared against each of the other renderings using the "compare two songs" feature in the song analysis software; as a result, each data point shown in this graph represents the mean of 91-300 comparisons of a total of 21 birds recorded at the ages shown. Error bars indicate SEMs.

$p=0.49$ ). The former two kinds of scores were $>0.80$ in all individuals, and many were at or near 1.00 , the score for a perfectly linear and consistent song. We infer that during any one recording period the three parameters measured did not discriminate between the songs of young and old adults that, to this extent, showed comparable stereotypy. Results provided by the more comprehensive stereotypy index, using the song analysis algorithm, are shown in Figure 4. Here again the stereotypy of motifs sung by young and old adults was comparable. A linear regression on these data showed no significant relationship between the age at recording and stereotypy $\left(F=2.43 \times 10^{-7} ; r=\right.$ $\left.1.1 \times 10^{-4} ; p=0.99\right)$. We did not follow individual birds longitudinally to see whether birds that had relatively low stereotypy scores as old adults also had relatively low scores as young adults. 


\section{Young}

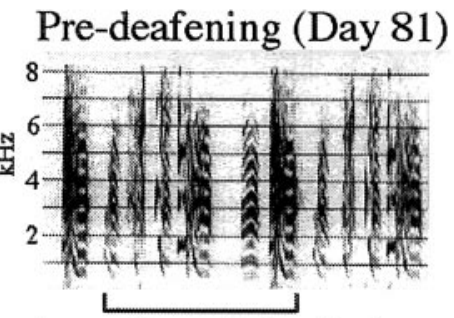

1 week post-deafening

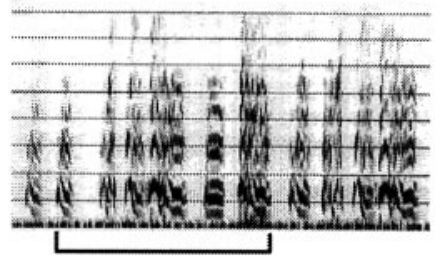

2 weeks post-deafening

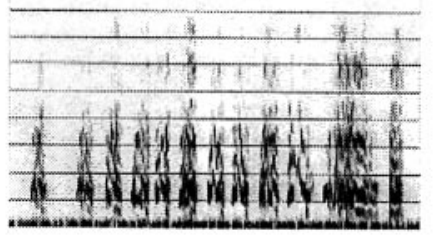

\section{5 weeks post-deafening}

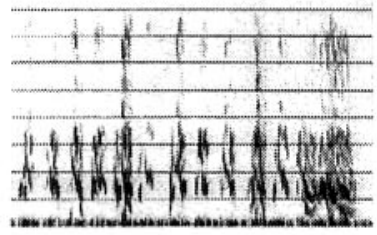

14 weeks post-deafening

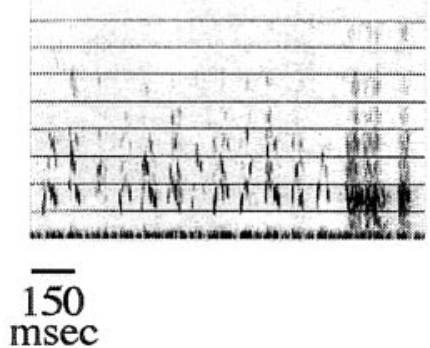

Middle-aged

Pre-deafening (Day 175)

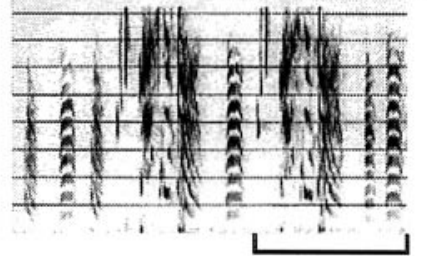

2 weeks post-deafening

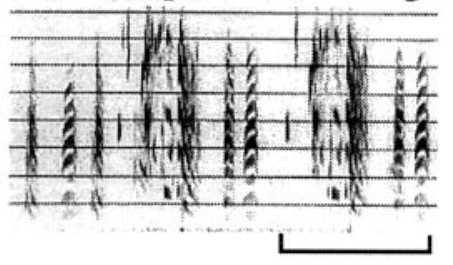

10 weeks post-deafening

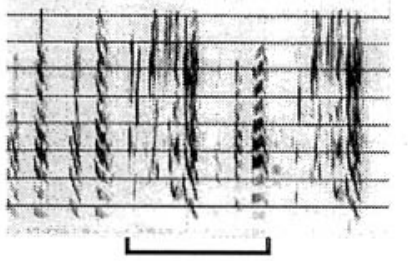

35 weeks post-deafening

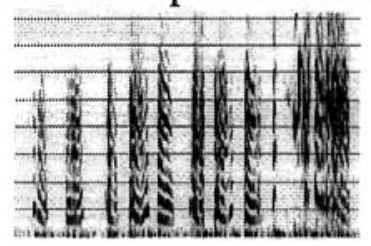

59 weeks post-deafening

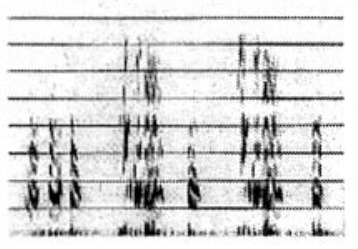

Old

Pre-deafening (Day 2090)

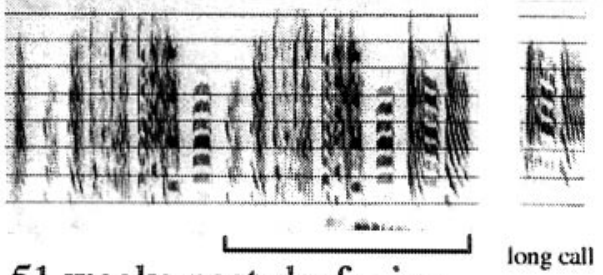

51 weeks post-deafening

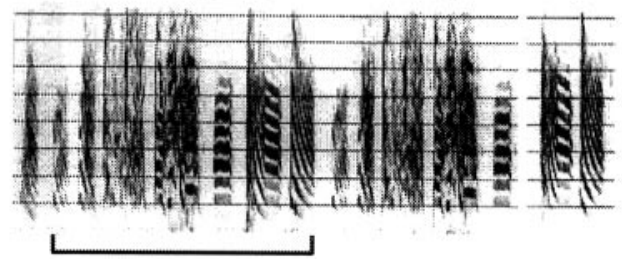

76 weeks post-deafening

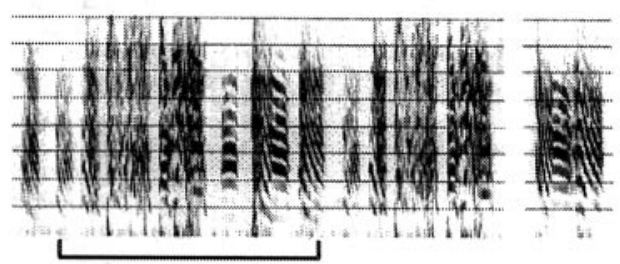

99 weeks post-deafening

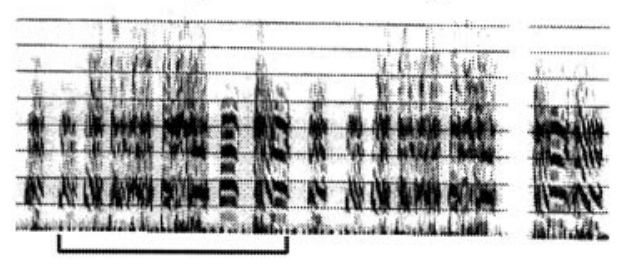

Figure 5. Sound-spectrographic representations of song degradation in three birds, deafened at $81 \mathrm{~d}$ (Young), $175 \mathrm{~d}$ (Middle-aged), and $2090 \mathrm{~d}($ Old) after hatching. Degradation of the long call is shown for two of these birds (Young, Old). The time after deafening is indicated for each of the songs. A continuous horizontal line is below the most complete rendering of each bird's song motif before deafening and after deafening for as long as that motif can be easily recognized. Notice that eventual degradation occurs in all three birds, but the onset, extent, and time course differs with the age at deafening. This observation, scored for all 16 birds in this experiment, is shown below (see Fig. 6). For purposes of comparison (this figure, see Fig. 6), the SSI for the last recording of the young, middle-aged, and old birds shown in this figure was $0.0,0.6$, and 2.8 , respectively.

\section{Effects of age at deafening on subsequent song degradation}

Song and long-call degradation after removal of both cochleae was very sensitive to the age of the bird at the time it was operated on (Fig. 5). In the youngest of these birds [young adults $(n=6)$, deafened on days 81-137], deterioration was already evident 1 week after surgery, and by 2-4 weeks the motif was barely recognizable; by 14 weeks after surgery song was typically a series of variable, degraded, poorly structured sounds that bore relatively little resemblance to the original, learned song. Birds that were middle-aged when deafened ( $n=3$; surgery on days 169231) showed slower song deterioration; in these birds the original 


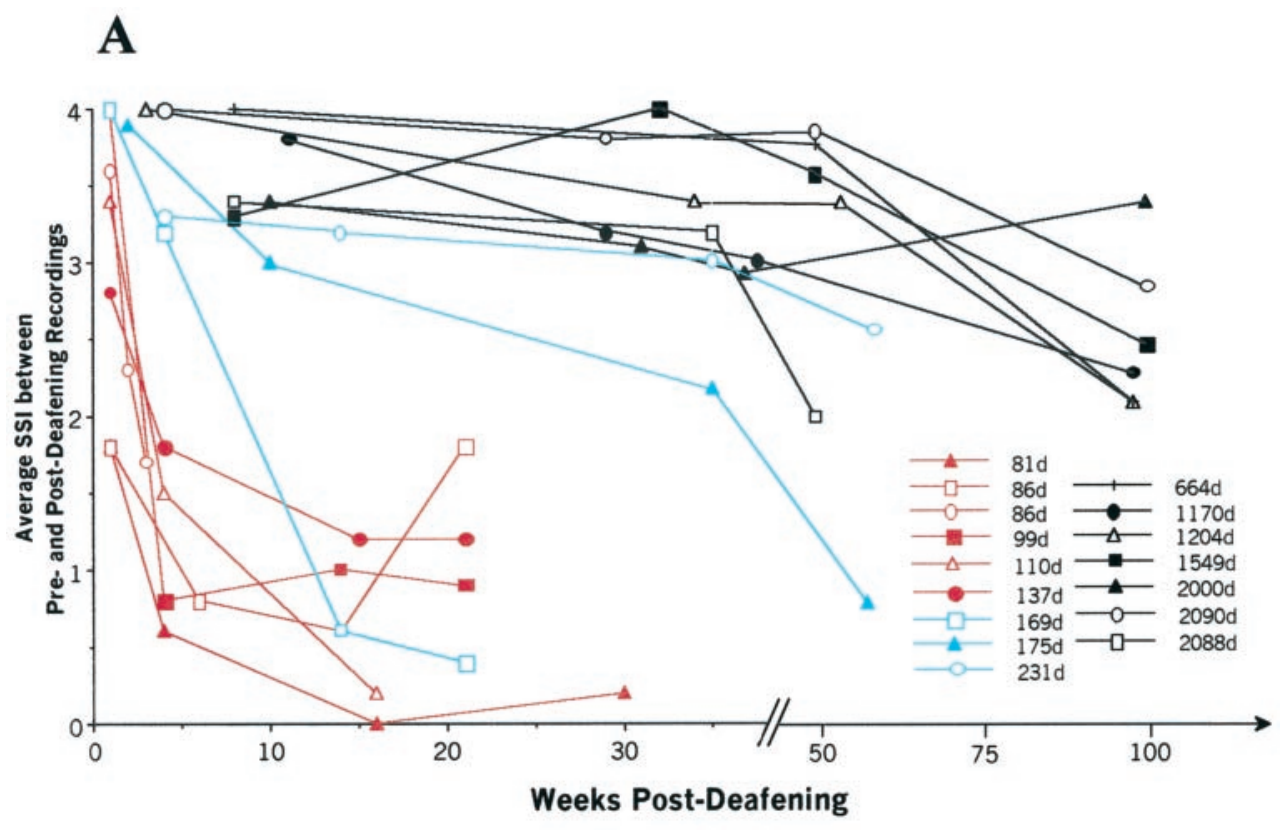

B

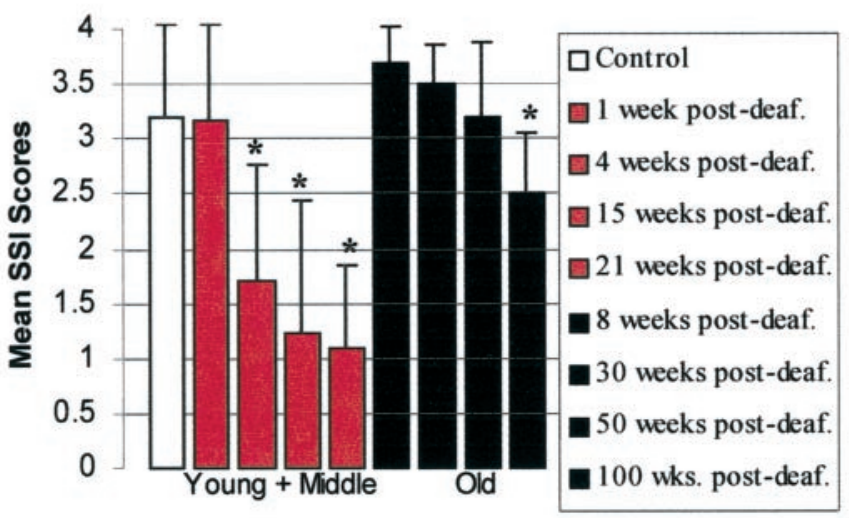

Figure 6. A, Song similarity index scores for all birds in this experiment plotted against time after deafening. Colors are used to identify adults that were young (red), middle-aged (blue), or old (black) at the time of deafening. Notice that song degradation, indicated by a declining similarity index, was greater and occurred faster in the birds deafened at the younger adult ages; birds deafened when they were older showed a slower decline. $B$, Mean similarity index scores for intact controls (white bar) and for the different postsurgery intervals for birds deafened as young or middle-aged adults (red bars) or as old adults (black bars). Birds in the two experimental groups were recorded at specified intervals after deafening, as shown in the box to the right of the figure. In each group's histogram, these intervals become longer from left to right, corresponding to the top to bottom listing inside the box. The mean control score is very conservative in that it compared songs from the same individual recorded 2-5 years apart. Error bars indicate the SEM. An asterisk indicates significance at the level of $p<0.02$ or better with respect to controls. Deaf., Deafening.

song motif was still recognizable at 10 weeks after surgery. At longer intervals, song and call deterioration was comparable in the birds deafened as young adults and middle-aged adults, although the older of the middle-aged birds showed a milder and slower effect (Fig. 6A).

Song deterioration was dramatically slower in birds deafened as old adults ( $n=7$; surgery on days 664-2090). These birds retained syllable structure and order in an easily recognizable manner even 51 weeks after deafening (Figs. 5, 6). Six of the birds in the oldest group continued to be recorded until 100-110 weeks after deafening. By that time, deterioration of the acoustic features of song was more advanced, but even then overall motif structure and the identity and sequential order of preoperative syllables and chunks were still recognizable. Figure $6 A$ captures these time trends as SSIs for all birds in the study. The three age groups we have defined are indicated by the color of the curves. By these scoring methods, song deterioration in the older birds proceeded at a rate that was $\sim 20$ times slower than that seen in the youngest ones. For statistical analysis, we compared the SSI scores of birds in the old group with the SSI scores of six intact controls; we also compared the SSI scores of the young and middle-aged birds, combined into one group, with the SSI scores of the intact controls. The young and middle-aged birds were not significantly different from controls at 1 week after deafening $(U=27.5 ; p<0.1)$ but were so at 4 weeks after deafening $(U=$ $35.5 ; p<0.05)$ and each time point thereafter. By contrast, the 


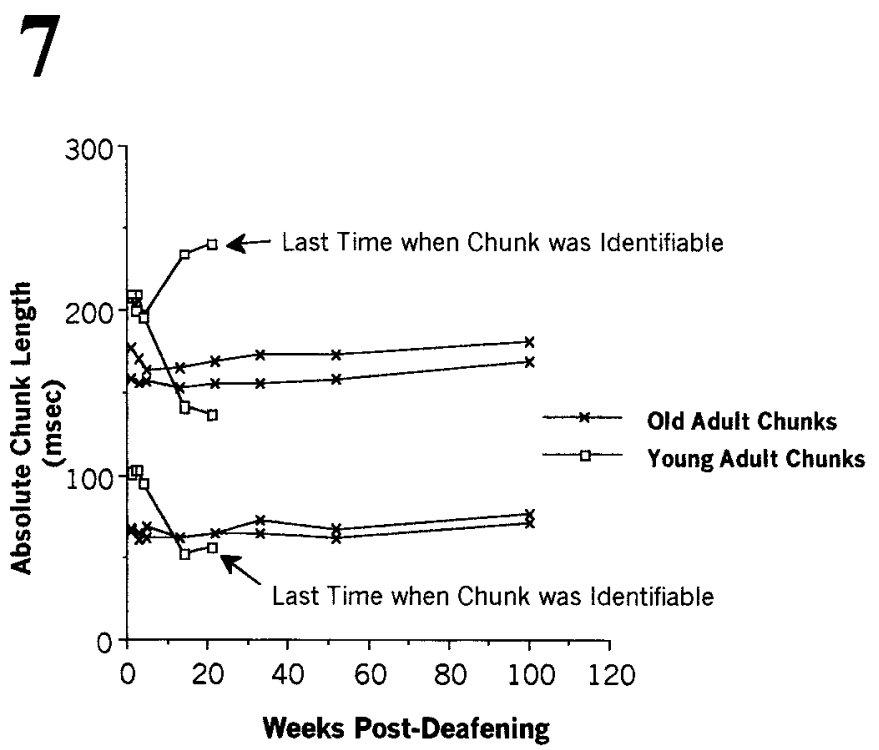

Figure 7. Postdeafening changes in chunk duration (in milliseconds) in one young adult and one old adult. The first data point in each series is the presurgery chunk duration; thus all points are shifted by 1 week. In these examples, the young bird had three chunks; the older bird had four. Notice that chunk duration changed more in the younger than in the older adult and that when changes occurred, they could be in the direction of making a chunk longer or shorter. The slope of regression lines fitted to each bird's performance during the first 8 postoperative weeks was calculated and used to produce the next figure (see Fig. 8).

old group was not significantly different from controls at 50 weeks after deafening $(U=22.5 ; p>0.1)$ but reached statistical difference at 100 weeks after deafening $(U=31 ; p=0.02)$. Figure $6 B$ shows a graph of these data for the control and deafened birds. In all cases the SSI scores of the intact controls corresponded, as described in Materials and Methods, to comparisons spanning $2-5$ years.

\section{Chunk duration is more resistant to deafening in older than in young birds}

Temporal parameters of the motif often persisted, in birds deafened at all ages, even as syllable structure deteriorated, as shown in Figure 5. Figure 7 shows, as an example of the fine grain quality of our data, chunk durations measured at different intervals after deafening in one bird that had been operated on as a young adult and in one that had been operated on as an old adult. Notice that the changes in chunk duration could be in the direction of making the chunk longer or shorter and that these changes were much more marked in the younger than in the older bird. We used a linear regression to derive a best-fit line for changes in duration for each chunk plotted in the manner of Figure 7 during the first 8 weeks after deafening. Taking the absolute value of the slopes of these best-fit lines and averaging them across all chunks for each bird provide a single measure of change in chunk duration after deafening. Our results for all birds show that over this time period chunk duration persisted much better in the older than in the younger birds (Fig. 8). In addition, chunk duration in the older birds persisted with little change for up to 2 years after deafening (e.g., Fig. 7), when recordings were discontinued.

\section{Effects of deafening on overall song structure}

The song of all the zebra finches deafened in this study retained its basic form of introductory notes followed by a more or less
8

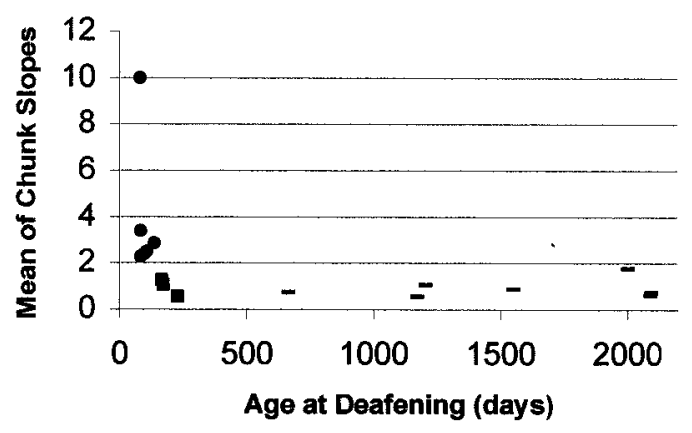

Figure 8. "Chunk slopes," in milliseconds per week, were determined by the absolute values of changing chunk duration (see Materials and Methods). These slopes reflect changes in chunk duration during the first 8 weeks after deafening; the larger the magnitude of the slope, the more chunk duration changed. Each bird is represented by a single symbol, and the code is as follows: $\boldsymbol{Q}$, young adult $(n=6) ; \mathbf{\square}$, middle-aged adult $(n=$ 3 ); and -, old adult $(n=7)$. The slope of the young birds is consistently above that of the other two age groups. The highest value corresponds to a bird deafened at $81 \mathrm{~d}$ of age.

reproducible motif, even when the song syllables were very degraded and the motif considerably shortened. The number of introductory notes increased in several birds. In one of the old birds (deafened at 2088 d) a silent interval was added in the middle of the song that then gave the impression that the bird was singing two motifs and that it alternated between the two. This change lowered that bird's SSI score at 50 weeks after deafening (Fig. 6A). However, even with these peculiarities, deafened birds retained the general plan of a bout that commenced with introductory notes followed by a motif.

Motifs that, as a result of deafening, had undergone marked changes were nonetheless surprisingly - although not completely, which would have precluded change - stable on any one day. This point is made by Figure 9, which shows examples of preoperative and postoperative song of the same bird; this bird was deafened at $1170 \mathrm{~d}$ of age, and the postoperative song was recorded 94 weeks after deafening. Although the motif recorded at the latter time had undergone marked degradation and had lost some of its syllables, it was remarkably stable in its renderings on any one day. This stability that persists after deafening differs from the instability (e.g., stuttering and addition or omission of syllables) induced by other forms of feedback perturbation (Leonardo and Konishi, 1999).

\section{Effects of deafening on the amount of singing}

We did not quantify the amount of directed and undirected song that occurred in our different experimental groups. However, we could not help but notice that, even though there were marked individual differences in the amount of singing when the birds were recorded, it was not the case that the groups deafened at older or younger adult ages subsequently sang more or less readily. Moreover, some individuals in both the young adult and old adult groups sang a lot or sang relatively little; yet the two groups showed no overlap in the time course of song deterioration, which was very homogeneous within each of these groups. 


\section{Pre-deafening}

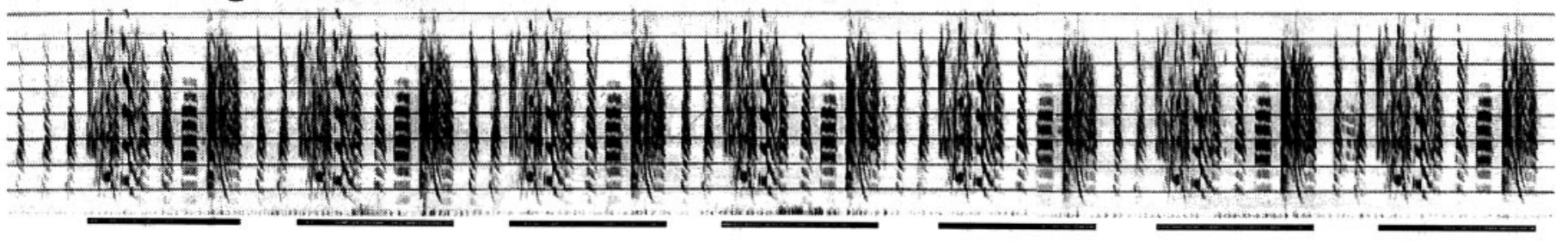

\section{4 weeks post-deafening}
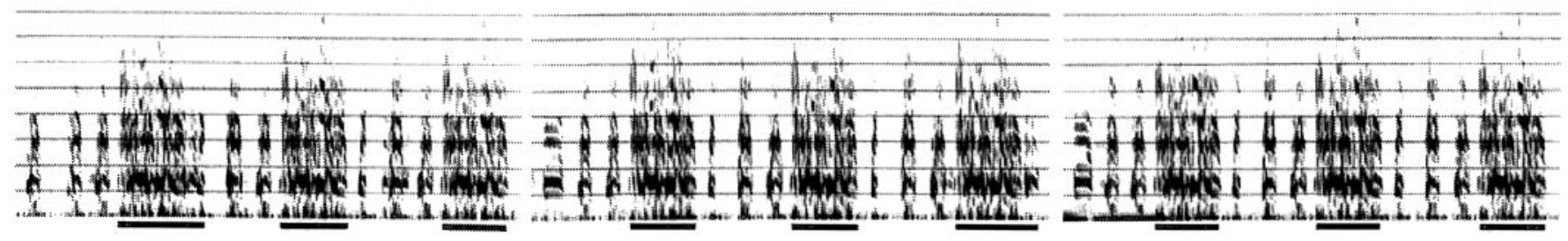

Figure 9. Bouts of directed song from an adult bird deafened at $1170 \mathrm{~d}$ recorded before (top) and 94 weeks after (bottom) deafening. The repeated unit is underlined in each case. Notice that although the degraded song (bottom) included a motif that was shorter than its preoperative version, it was nonetheless repeated in a rather consistent manner. Consistency in the production of a degraded song was typical of most postoperative recordings.

\section{Results of a two-step deafening operation: a control for the surgical procedure}

One cochlea of a male zebra finch was removed on day 86 . The song this bird produced during the ensuing 16 weeks showed no deterioration (Fig. 10A), suggesting that the surgery itself did not result in song changes and that a single cochlea was sufficient to maintain song. The second cochlea was removed on day 231 . There was a relatively mild deterioration of song during the next 60 weeks (Fig. 10B), as might have been expected from a bird that was deafened (removal of second cochlea) at an age that fell between that of our two extreme age groups; the results of this bird's second operation are part of the graph in Figure 6 (the individual deafened at $231 \mathrm{~d}$ ).

\section{DISCUSSION}

We show that the stereotypy of adult male zebra finch song is very similar between the ages of $90 \mathrm{~d}$ and 5 years. Young and old adults yielded similar scores for linearity and consistency of syllable transitions, stability of chunk duration, and stability in the acoustic features of successive repetitions of a same motif.

Song deterioration after deafening, on the other hand, was highly correlated with the age at deafening, at least during the first 2 years after surgery. The song of individuals deafened at 3 months of age started to deteriorate 1 week later, the song of birds deafened at 5-6 months showed a slower onset of deterioration, and that of individuals deafened at 2-5 years of age took a year or longer to show measurable changes. The middle-aged group in this comparison, although small, shows greater individual variability in outcome than the other two. Interestingly, previous studies that failed to see the age-related effect used birds ranging in age from 4 to 10 months (Price, 1979; Bottjer and Arnold, 1984; Nordeen and Nordeen, 1992), comparable with our middle-aged group. We have no evidence that the effect of age at deafening was caused by differences in how much the birds in the different groups sang after they were deafened. We infer that neither the degree of song stereotypy before surgery nor the amount of singing after surgery accounted for the age-dependent effect of deafening on song. The variables responsible for the latter effect must therefore be sought in other events that occurred before surgery.

Our results can be explained by two kinds of hypotheses. Hypothesis 1 would argue that the persistence of a learned motor pattern (song) is determined, after deafening, by the number of times that pattern had been produced by the hearing bird. Hypothesis 2 would argue that the persistence of this pattern is determined not by how much the bird has sung but by the age at deafening. The time when song was learned was not a variable because all our birds probably acquired their song before they reached sexual maturity, at $\sim 80 \mathrm{~d}$. We will now evaluate the possible contributions of experience and age.

\section{Hypothesis 1: the effect of experience}

The benefits of practice for quality of performance in sports, music, and ballet are well known and have been presented in many guises (Gallwey, 1974; Asanuma and Pavlides, 1997). In principle, the same benefits could apply to adult male zebra finches. Our birds had many opportunities to sing, encouraged by the social setting in which they were kept. Zebra finches sing throughout the year under laboratory conditions and will continue to sing their normal song even after castration (Arnold, 1975b). We do not know how many times each bird sang before deafening, but 20 songs per day after sexual maturity is probably a very conservative estimate. If we accept this estimate as a minimum, then individuals in our group of old adults would have sung their song at least 13000-42000 times before deafening. The youngest birds, on the other hand, even if they had crystallized their song as early as $70 \mathrm{~d}$ after hatching, and sang as many as 100 songs per day, would have sung at most 1000 or so songs before deafening. There is no reason to think that zebra finches sing less as they age, at least over the span of ages represented in our birds (our personal observations). We infer that our age groups differed systematically in how many times birds in them had sung before deafening, with many more songs having been produced by the birds deafened at the older ages. It should be noted, however, that if the number of songs produced before deafening was the determining variable, then there must have been a ceiling effect be- 
A

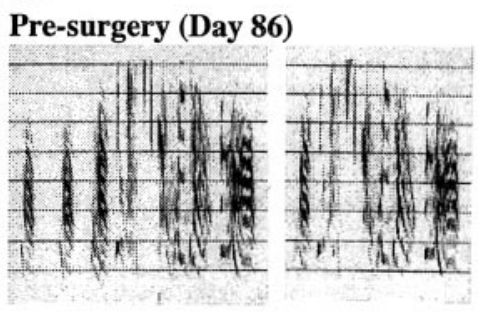

B

4 weeks after second cochlea removed (Day 231)
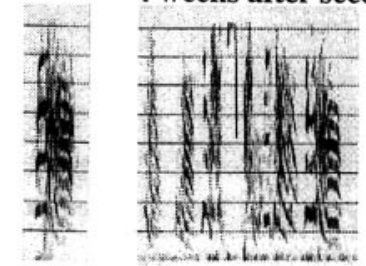

14 weeks after second cochlea removed

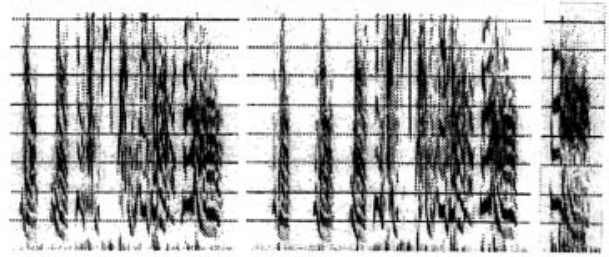

60 weeks after second cochlea removed

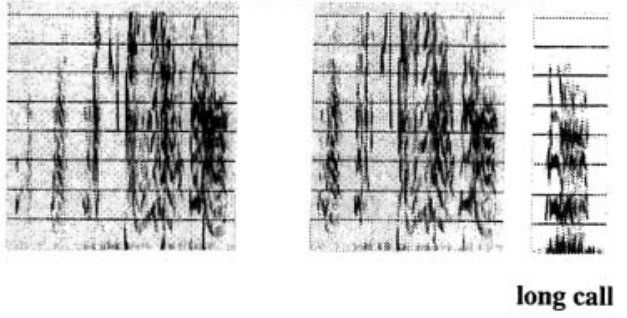

Figure 10. Results of a deafening operation done in two steps. $A$, The left cochlea was removed when the bird was $86 \mathrm{~d}$ old. $B$, The right cochlea was removed at $231 \mathrm{~d}$ of age, which falls between the age ranges of the middle-aged and old birds shown in Figure 5. The time after each surgery is shown for each recording. Notice that there were no song changes after the first operation but song degraded after the second operation at a rate intermediate between what would have been expected from the middle-aged and old birds shown in Figure 5. (This bird is represented on the graph in Fig. 6 where its age at deafening, $231 \mathrm{~d}$, corresponds to that when the second cochlea was removed.) This bird's long call appears at the end of the song motif. Although the long call was unaffected by the first operation, it too had deteriorated by 60 weeks after removal of the second cochlea. The asterisk at the bottom in $A$ marks the long call of an unseen female zebra finch used to elicit long-call responses from the male. This bird's single cochlea permitted him to hear, process, and respond to this female's long call at 16 weeks after surgery.

cause there was no obvious age trend in the birds deafened at $2-5$ years of age.

We suggest that many repetitions of a stable, learned motor pattern, with unimpeded access to auditory feedback, result in a form of learning that we will call "engrainment." Engrainment could result from the repeated, normal confluence of a motor output and its resulting auditory and nonauditory feedback. However, although there is ample evidence of the role of auditory feedback in song acquisition and maintenance, there is no such evidence of the role of nonauditory (proprioceptive?) feedback (Bottjer and Arnold, 1984). Engrainment was first used in the field of human psychology to refer to "the process by which existing connections among familiar information are strengthened when the information is retrieved or utilized" (MacKay and Burke, 1990). The engrainment hypothesis suggests that we are dealing with a process in which, as practice continues, the circuits that produce learned song become increasingly stable over long periods of time. This stability could free these circuits from dependence on auditory feedback and its presumed role in error correction. A glimmer of hypothesis 1 was offered in previous work done with chaffinches, but the database was so limited as to be anecdotal [Nottebohm (1968), my Fig. 6].

\section{Hypothesis 2: the effect of age}

Older individuals are usually not credited with having superior memory, yet it may be fruitful to contemplate the following scenario. We know that neurons are constantly added to parts of the song system of songbirds (Goldman and Nottebohm, 1983; Paton and Nottebohm, 1984). We also know that this addition peaks during ontogeny and at times of the year when song learning occurs (Alvarez-Buylla and Nottebohm, 1988; Nordeen and Nordeen, 1988; Alvarez-Buylla et al., 1990). We know, too, that neurons added in adulthood replace others that have died previously (Kirn and Nottebohm, 1993; Kirn et al., 1994; Scharff et al., 2000). It has been suggested that such turnover facilitates learning, even though it may also compromise earlier memories (Kirn et al., 1994). If neuronal turnover in adult male zebra finches were to decrease with age - a matter on which we have no information but that was suggested by observations in canaries (Alvarez-Buylla et al., 1990)—-then a possible consequence of this could be that learned song memories would be made more stable in the older individuals and hence, perhaps, more resistant to deafening. Because there is, yet, no information about agedependent neuronal turnover in adult zebra finches, we cannot evaluate the plausibility of this hypothesis.

A variant of the age-dependent survival of memory would be that the effect of deafening on the survival of song system neurons (Wang et al., 1999), including those of nucleus high vocal center, decreases with age, which could explain why birds deafened at older ages retain their preoperative song for longer periods of time. Age could, of course, affect the stability of song pathways in 
still other ways, but for reasons of space we will not explore these possibilities.

We are aware that the effects of experience and age could interact, in which case the outcome we observed could be dependent on both singing experience and age. We would like to emphasize, too, that our data on song deterioration should not be taken as final proof that a song memory has been lost. The motor program could still be there, intact, yet access could be incomplete or faulty. However, we suggest that when deterioration of performance is gradual and steady and leads to the eventual disappearance of learned structure, then the most parsimonious interpretation is that the motor program or memory also has undergone gradual deterioration and that for it to occur once more it would have to be relearned.

If hypothesis 1 is validated, then song learning in oscine songbirds occurs in four stages: (1) sounds heard are memorized as a model, (2) this model and auditory feedback guide vocal development, (3) auditory feedback is used to maintain the learned vocal pattern, and (4) repetition of the pattern thus maintained engrains the motor act, which becomes more and more independent of auditory feedback. If one accepts engrainment as a form of learning, then learning-related changes in gene expression, cell morphology, or the number of cells drawn into a circuit need not be restricted to ontogeny, when the pattern is first acquired, but may occur every time the bird sings. As was suggested in a previous publication (Jarvis et al., 1995), finding behavioral situations when learning does not occur may prove difficult.

W. H. Thorpe (1958) was the first to refer to the achievement of adult song stereotypy by a young songbird as a process of song "crystallization." Our results suggest that although behavioral crystallization, i.e., the change from a variable to a stable pattern, often occurs over a period of time measured in days or weeks, the permanence of the underlying motor memory may continue to increase for many months thereafter. We propose that the term crystallization be used to refer to the achievement of adult song stereotypy and that the term engrainment, if confirmed as a phenomenon, be reserved for the protracted, additive, experience-dependent growth of a memory's stability. Behavioral stability is no guarantee of memory stability.

Our results do not discriminate between hypotheses 1 and 2-and one could think of others-but they are testable. Regardless of which one is right, the fact remains that learned song in male zebra finches becomes more resistant to deafening as months and years go by, and the song system of old adult zebra finches may show important differences from that of young adults. As these differences are revealed, we will better understand the lifelong process of song maintenance.

\section{REFERENCES}

Alvarez-Buylla A, Nottebohm F (1988) Migration of young neurons in adult avian brain. Nature 335:353-354.

Alvarez-Buylla A, Kirn JR, Nottebohm F (1990) Birth of projection neurons in adult avian brain may be related to perceptual or motor learning. Science 249:1444-1446.

Arnold A (1975a) The effects of castration on song development in zebra finches (Poephila guttata). J Exp Zool 191:261-278.

Arnold A (1975b) The effects of castration and androgen replacement on song, courtship, and aggression in zebra finches (Poephila guttata). J Exp Zool 191:309-325.

Asanuma H, Pavlides C (1997) Neurobiological basis of motor learning in mammals. NeuroReport 8:i-vi.

Bischof HJ, Böhner J, Sossinka R (1981) Influence of external stimuli on the quality of the song of the zebra finch (Taeniopygia guttata castanotis Gould). Z Tierpsychol 57:261-267.
Böhner J (1990) Early acquisition of song in the zebra finch, Taeniopygia guttata. Anim Behav 39:369-374.

Böhner J, Harding C, Marler P (1992) Androstenedione therapy reinstates normal, not supernormal, song structure in castrated adult male zebra finches. Horm Behav 26:136-142.

Borden GJ (1979) An interpretation of research on feedback interruption in speech. Brain Lang 7:307-319.

Bottjer SW, Arnold AP (1984) The role of feedback from the vocal organ. I. Maintenance of stereotypical vocalizations by adult zebra finches. J Neurosci 4:2387-2396.

Cynx J (1990) Experimental determination of a unit of song production in the zebra finch (Taeniopygia guttata). J Comp Psychol 104:3-10.

Eales LA (1985) Song learning in zebra finches: some effects of song model availability on what is learnt and when. Anim Behav 33:1293-1300.

Eales LA (1987) Song learning in female-raised zebra finches: another look at the sensitive phase. Anim Behav 35:1356-1365.

Gallwey WT (1974) The inner game of tennis. New York: Random House.

Goldman S, Nottebohm F (1983) Neuronal production, migration, and differentiation in a vocal control nucleus of the adult female canary brain. Proc Natl Acad Sci USA 80:2390-2394.

Gould JL, Marler P (1987) Learning by instinct. Sci Am 255:74-85.

Immelmann K (1969) Song development in the zebra finch and other estrildid finches. In: Bird vocalizations (Hinde RA, ed), pp 61-74. Cambridge: Cambridge UP.

Jarvis E, Mello CV, Nottebohm F (1995) Associative learning and stimulus novelty influence the song-induced expression of an immediateearly gene in the canary forebrain. Learn Mem 2:62-80.

Kelley DB, Nottebohm F (1979) Projections of a telencephalic auditory nucleus-field L-in the canary. J Comp Neurol 183:455-470.

Kirn J, Nottebohm F (1993) Direct evidence for loss and replacement of projection neurons in adult canary brain. J Neurosci 13:1654-1663.

Kirn J, O'Loughlin B, Kasparian S, Nottebohm F (1994) Cell death and neuronal recruitment in the high vocal center of adult male canaries are temporally related to changes in song. Proc Natl Acad Sci USA 91:7844-7848.

Konishi M (1964) Effects of deafening on song development in two species of juncos. Condor 66:85-102.

Konishi M (1965) The role of auditory feedback in the control of vocalization in the white-crowned sparrow. Z Tierpsychol 22:770-783.

Leonardo A, Konishi M (1999) Decrystallization of adult birdsong by perturbation of auditory feedback. Nature 399:466-470.

MacKay DG, Burke DM (1990) Cognition and aging: a theory of new learning and the use of old connections. In: Aging and cognition: knowledge organization and utilization. Amsterdam: Elsevier.

Marler P (1970) Birdsong and speech: could there be parallels? Am Sci 58:669-673.

Marler P, Nelson D (1993) Measurement of song learning behavior in birds. In: Paradigms for the study of behavior (Conn MP, ed), pp 447-465. San Diego: Academic.

Morris D (1954) The reproductive behavior of the zebra finch (Poephila guttata), with special reference to pseudofemale behavior and displacement activities. Behavior 6:271-321.

Morrison R, Nottebohm F (1993) Role of a telencephalic nucleus in the delayed song learning of socially isolated zebra finches. J Neurobiol 24:1045-1064.

Nordeen KW, Nordeen EJ (1988) Projection neurons within a vocal motor pathway are born during song learning in zebra finches. Nature 334:149-151.

Nordeen KW, Nordeen EJ (1992) Auditory feedback is necessary for the maintenance of stereotyped song in adult zebra finches. Behav Neural Biol 57:58-66.

Nottebohm F (1968) Auditory experience and song development in the chaffinch, Fringilla coelebs. Ibis 110:549-568.

Nowicki S, Nelson DA (1990) Defining natural categories in acoustic signals: comparison of three methods applied to "chick-a-dee" call notes. Ethology 86:89-101.

Paton JA, Nottebohm F (1984) Neurons generated in the adult brain are recruited into functional circuits. Science 225:1046-1048.

Price P (1979) Developmental determinants of structure in zebra finch song. J Comp Physiol Psychol 93:260-277.

Scharff C, Nottebohm F (1991) A comparative study of the behavioral deficits following lesions of various parts of the zebra finch song system: implications for vocal learning. J Neurosci 11:2896-2913. 
Scharff C, Kirn JR, Grossman M, Macklis JD, Nottebohm F (2000) Targeted neuronal death affects neuronal replacement and vocal behavior in adult songbirds. Neuron 25:481-492.

Simpson HB, Vicario DS (1990) Brain pathways for learned and unlearned vocalizations differ in zebra finches. J Neurosci 10:1541-1556.

Sossinka R, Böhner J (1980) Song types in the zebra finch Poephila guttata castanotis. Z Tierpsychol 53:123-132.

Tchernichovski O, Nottebohm F, Ho CE, Pesaran B, Mitra PP. (2000) A procedure for an automated measurement of song similarity. Anim Behav, in press.

Thorpe W (1958) The learning of song patterns by birds, with especial reference to the song of the chaffinch, Fringilla coelebs. Ibis 100:535-570.

Volman S, Khanna H (1995) Convergence of untutored song in groupreared zebra finches. J Comp Psychol 109:211-221.

Waldstein R (1990) Effects of postlingual deafness on speech produc- tion: implications for the role of auditory feedback. J Acoust Soc Am 88:2099-2114.

Walters M, Collado D, Harding C (1991) Oestrogenic modulation of singing in male zebra finches: differential effects on directed and undirected songs. Anim Behav 42:445-452.

Wang N, Aviram R, Kirn JR (1999) Deafening alters neuron turnover within the telencephalic motor pathway for song control in adult zebra finches. J Neurosci 19:10554-10561.

Williams H (1990) Models for song learning in the zebra finch: fathers or others? Anim Behav 39:747-757.

Williams H, Staples K (1992) Syllable chunking in zebra finch (Taeniopygia guttata) song. J Comp Psychol 106:278-286.

Zann R (1985) Ontogeny of the zebra finch distance call. I. Effects of cross-fostering to Bengalese finches. Z Tierpsychol 68:1-23.

Zann R (1993) Structure, sequence, and evolution of song elements in wild Australian zebra finches. The Auk 110:702-715. 